\title{
Numerical Calculations of Some Plasma Parameters of the Capacitively Coupled $R F$ Discharge
}

\author{
Mohamed Ali Hassouba1,2, Ahmed Rida Galaly ${ }^{3,4^{*}}$, Usama Mohamed Rashed ${ }^{5}$ \\ ${ }^{1}$ Applied Physics Department, Faculty of Applied Sciences, Taibah University, Medina, KSA \\ ${ }^{2}$ Physics Department, Faculty of Sciences, Benha University, Qalyubia, Egypt \\ ${ }^{3}$ Engineering Science Department, Faculty of Community, Umm Al-Qura University, Mecca, KSA \\ ${ }^{4}$ Physics Department, Faculty of Sciences, Beni-Suef University, Beni-Suef, Egypt \\ ${ }^{5}$ Physics Department, Faculty of Sciences, Alazhar University, Cairo, Egypt \\ Email: ${ }^{*}$ ahmed galaly@yahoo.com
}

Received 12 February 2014; revised 11 March 2014; accepted 9 April 2014

Copyright ( 2014 by authors and Scientific Research Publishing Inc.

This work is licensed under the Creative Commons Attribution International License (CC BY).

http://creativecommons.org/licenses/by/4.0/

(c) (i) Open Access

\begin{abstract}
Numerical calculations by using a self-consistent model of the collisional sheath for the capacitively coupled $R F$ discharge are our target. The results indicated that, at high pressure, the ohmic heating is usually the dominant heating mechanism in the discharge. The power dissipated in the sheath is calculated and compared with the measured data. Moreover, we indicated that, when the gas pressure is increased, the calculated dissipated power is decreased also while the measured input $R F$ power is increased. Furthermore the sheath thickness of the capacitively coupled discharge is calculated and in the same order of the electron oscillation amplitude in the $R F$ field, while the ionization mean free path is shorter than it.
\end{abstract}

\section{Keywords}

\section{$R F$-Discharge, Self-Consistent Model, Ohmic Heating}

\section{Introduction}

Capacitively coupled $R F$ discharges were widely used in the microelectronics industry for the production of integrated circuits specially processes such as etching and coating [1]-[5]. In these systems, the metallic electrodes were normally enclosed within the reactor and in direct contact with the plasma. External conditions, like pres-

*Corresponding author. 
sure, power, geometry and driving frequency determine the properties of the plasma and thus its practical use in various applications [6].

When $R F$ voltage is applied between two parallel plate electrodes, two sheaths are formed in front of each electrode and plasma connecting these sheaths. These sheaths heat the electrons through a process in which electrons reflected from the moving sheath edge and gained energy on average. This process usually referred to Stochastic heating which is the dominant source of electron heating at low pressure besides the well-known ohmic heating. Heating by RF radiation was very important in the fusion device [7]. Two kinds of electron heating mechanisms may be taken place. Firstly, ohmic heating due to the transfer of energy gained from the acceleration of electrons in electric field to thermal electron energy through collisional processes. Secondly, Stochastic electron heating has been found to be a powerful mechanism in capacitive coupling R-F discharge. Here electrons impinging on the oscillating sheath edge suffer a change of velocity upon reflection back into the bulk plasma. As the sheath moves into the bulk, the reflected ions gain energy, as the sheath moves away, the electron loss energy. However, averaging over an oscillation period, there is a net energy gain.

At sufficiently large $R F$ voltage, the beams of fast electrons ensure the high rate of ionization in the quasineutral plasma. This ionization, as well as the diffusion of charged particles near the boundaries of the sheaths compensates the losses of electrons and ions in the central part of the discharge due to recombination and diffusion. The discharge becomes stable and continues to burn in the strong-current regime, and low-frequency oscillations disappear [8].

The aim of the present work is using a self-consistent analysis of the collisional sheath of the capacitively coupled $R F$ discharge to calculate the electron ohmic heating power, the average Stochastic power for a single sheath, the power dissipated in the sheath and the sheath thickness and compared the results with the available experimental data.

\section{Experimental Setup}

The capacitive discharge plasma was formed between two stainless-steel $20 \mathrm{~cm}$ diameter electrodes separated by $6 \mathrm{~cm}$. The lower electrode was powered by $R F(13.56 \mathrm{MHz})$, type ENI model OEM-6, generator through a matching box, while the upper electrode as well as the discharge chamber was grounded. The chamber was pumped down by a vacuum system to base pressure of $10^{-4}$ torr. During all measurements, continues flow of a gas through the discharge chamber was maintained. High purity Ar gas was used as the working gas and was fed to the chamber through a needle valve. The pressure of the working gas was varied between $1-10$ torr and measured using a digital vacuum gauge (VAP 5). For displaying the waveform of the discharge current and voltage two channel digital oscilloscope (Tektronix TDS 320, $100 \mathrm{MHz}$ ) was used. The applied voltage "V" was measured by using HF voltmeter type B7-37, while the discharge current "I" was measured from its waveform with the help of an oscilloscope. Bird Model 4431-43 Series wattmeter was used to measure the input and/or dissipated powers.

Figure 1 shows a schematic drawing of the apparatus used in this work. Also, Figure 2 shows an example of the waveform of $R F$ discharge at discharge voltage $=950 \mathrm{~V}$ and at Ar gas pressure $=1$ torr.

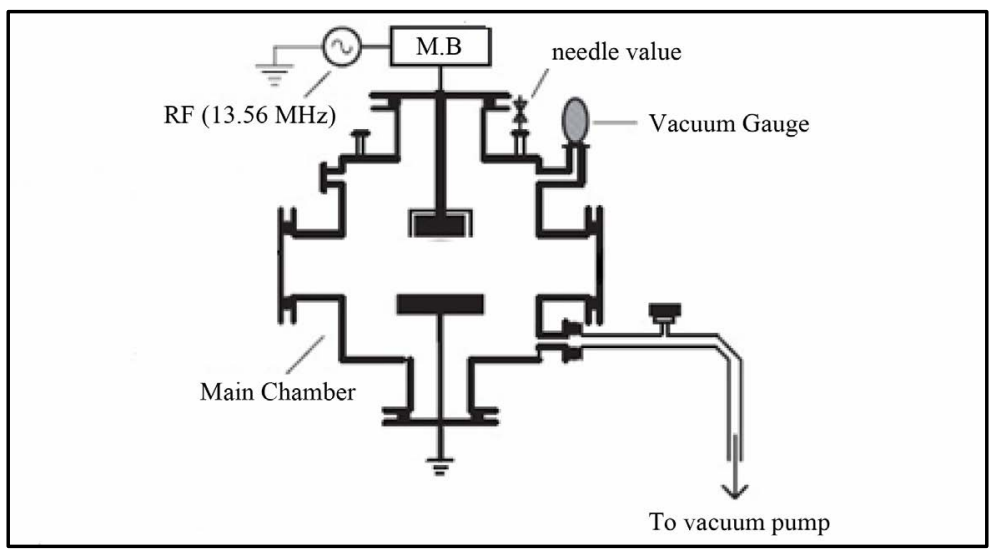

Figure 1. It shows a schematic drawing of the experimental setup. 


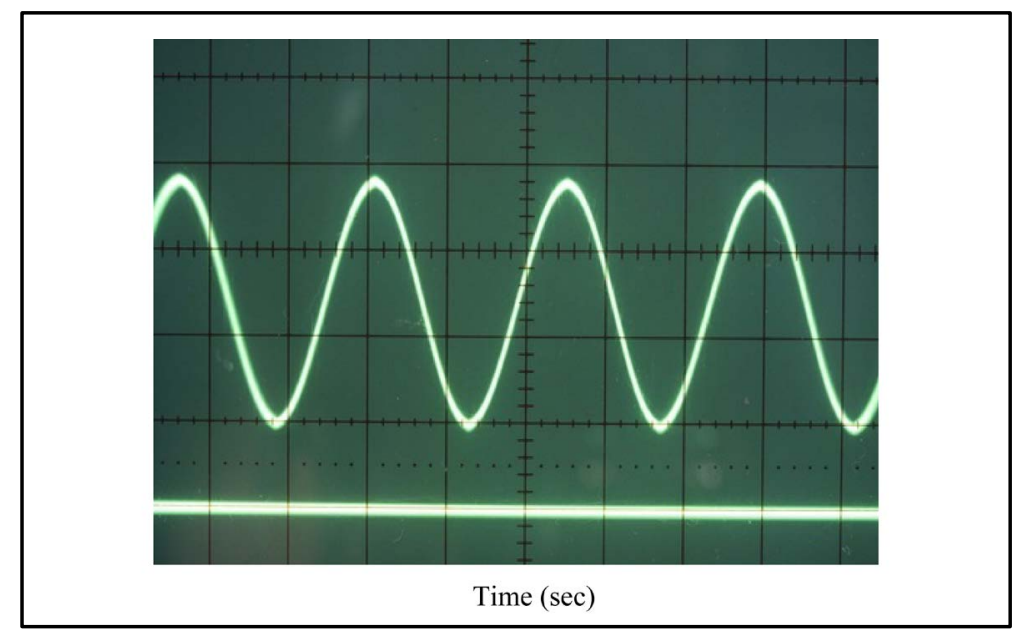

Figure 2. It shows an example of the waveform of $R F$ discharge at discharge voltage $=950 \mathrm{~V}$ and at Ar gas pressure $=1$ torr.

\section{Theoretical Considerations}

At high pressure a self-consistent analysis of the collisional sheath is required, which has been given by Liebermann [9] and Godyak [10]. These authors assuming that, the ion mean free path $\lambda_{i}$ is constant independent of the velocity [11]. Also, assuming the ionization occurs in the sheath, and the electrons are accelerated into the plasma.

\subsection{Self-Consistent Model}

The self-consistent model equations are summarized below. Assuming that the plasma length $d \approx l-2 S_{m}$ (where $S_{m}$ is the maximum sheath thickness) $l$ is the system length, with an initial estimate $S_{m} \approx 1 \mathrm{~cm}$ for numerical computations which is a nominal value for high-pressure capacitive $R F$ discharge. From particle conservations [12]:

$$
\frac{K_{\text {iz }}}{U_{B}}=\frac{1}{n_{g} d_{\text {eff }}}
$$

(where $K_{i z}$ is the rate constant for electron-neutral ionization, $U_{B}$ is the Bohm velocity, $n_{g}$ is the neutral atom density and $d_{e f f}$ is an effective plasma size).

At intermediate and low pressure, we have:

$$
\begin{aligned}
\frac{K_{i z}}{u_{s}}=\frac{1}{n_{g} d_{\text {eff }}} & =\frac{2}{n_{g} d} \frac{n_{s}}{n_{0}}, \quad \lambda_{i} \geq\left(\frac{T_{i}}{T_{e}}\right) d \\
\frac{n_{s}}{n_{0}}= & 0.86\left(3+\frac{1}{2 \lambda_{i}}\right)^{-1 / 2}
\end{aligned}
$$

( $n_{s}$ is the ion density at the plasma sheath edge, $n_{0}$ is the plasma density). Where $u_{s}=u_{B}$ for collisionless sheath and where at higher pressure one has:

$$
\frac{\left(K_{m i} K_{i z}\right)^{1 / 2}}{U_{B}}=\frac{\pi}{n_{g} d} \quad \lambda_{i} \leq\left(\frac{T_{i}}{T_{e}}\right) d
$$

where $K_{m i}$ is the ion-neutral momentum transfer rate constant.

And the $\left(n_{s} / n_{0}\right)$ ratio becomes:

$$
\frac{n_{s}}{n_{0}}=\left[1+\left(\frac{l}{\pi} \frac{U_{B}}{D_{a}}\right)^{2}\right]^{\frac{-1}{2}}
$$


where $D_{a}$ is the ambipolar diffusion coefficient. The electron ohmic heating power per unit area is given by:

$$
\begin{gathered}
S_{\text {ohm }}=1.73\left(\frac{m}{2 e} \frac{n_{s}}{n_{0}} \varepsilon_{0} \omega^{2} \vartheta_{m} d V_{1}^{1 / 2} T_{e}^{1 / 2}\right) \quad \lambda_{i} \geq\left(\frac{T_{i}}{T_{e}}\right) d \\
S_{\text {ohm }}=1.73\left(\frac{m}{2 e} \frac{n_{s}}{n_{0}} \varepsilon_{0} \omega^{2} \vartheta_{m} V_{1}^{1 / 2} T_{e}^{1 / 2} \frac{2}{\beta} \ln \tan \left[\frac{\pi}{4}+\frac{\beta d}{4}\right]\right) \quad \lambda_{i} \leq\left(\frac{T_{i}}{T_{e}}\right) d
\end{gathered}
$$

where $V_{1}=\left(V_{a b} / 2\right)$ is the fundamental $R F$ voltage amplitude across a single sheath, $v_{m}$ is the electron neutral frequency and $\beta$ is a constant equal to $(\pi / l)$.

Also, the average Stochastic power for a single sheath is given by:

$$
S_{\text {st }}=0.45\left(\frac{m}{e}\right)^{1 / 2} \varepsilon_{0} \omega^{2} T_{e}^{1 / 2} V_{1}
$$

The ion kinetic energy per ion hitting the electrode is

$$
E_{i}=V=0.83 V_{1}
$$

The electron power balance equation is

$$
S_{e}=S_{\text {ohm }}+2 S_{s t}=2 e n_{s} U_{s}\left(E_{c}+2 T_{e}\right)
$$

So, the total power absorbed per unit area is then found as:

$$
S_{a b s}=2 e n_{s} U_{s}\left(V+E_{c}+2 T_{e}\right)
$$

Eliminating $n_{s}$ from Equations (10), (11) and using Equation (9) for $V$, one can obtain the final equation as:

$$
S_{a b s}=\left(S_{o h m}+2 S_{s t}\right)\left(1+\frac{0.83 V_{1}}{E_{c}+2 T_{e}}\right)
$$

Take the following parameters:

$p=1$ torr (Ar gas used), $l=10 \mathrm{~cm}$,

$f=13.56 \mathrm{MHz} \rightarrow \omega=8.6 \times 10^{7} \mathrm{~Hz}, V_{R F}=930 \mathrm{~V}, T_{e}=8 \mathrm{eV}$,

$n_{g}=3.55 \times 10^{22} \mathrm{~m}^{-3}, k_{i z}=7 \times 10^{-15} \mathrm{~m}^{3} / \mathrm{sec}$,

$k_{n i}=1.4 \times 10^{-14} \mathrm{~m}^{3} / \mathrm{sec}$ and $k_{e l}=1.6 \times 10^{-13} \mathrm{~m}^{3} / \mathrm{sec}$,

Starting with $S_{m}=1 \mathrm{~cm}, \lambda_{i}=3 \times 10^{-3} \mathrm{~cm}, d=1-2 S_{m}=8 \mathrm{~cm}$.

$\therefore \lambda_{i} \ll d$, which is in the high pressure region, in which the plasma is relatively curved in the center. Also, the $R F$ electric field rises at the plates and falls at the mid plane.

Substituting into Equations (8), (9) we get:

$$
S_{\text {ohm }}=118 \mathrm{~W} / \mathrm{m}^{2}, \quad S_{s t}=77 \mathrm{~W} / \mathrm{m}^{2} .
$$

This result indicates that, at high pressure, the ohmic heating is usually the dominant heating mechanism.

\subsection{Power Dissipation in the Sheath}

The power $P_{s}$ dissipated in the sheath is given by the product of the time averaged sheath voltage $V_{p}$ and the ion current $I_{R}$.

$$
P_{s}=V_{p} \cdot I_{R}=\left|V_{p}\right|\left|I_{R}\right|
$$

After expressing $V_{p}$ by the Child-Langmuir law: where

$$
\left|V_{p}\right|=\frac{3}{5}\left(\frac{3\left|I_{R}\right| \sqrt{p}}{2 \varepsilon_{0} A k}\right)^{2 / 3} d_{S}^{5 / 3}
$$

$p$ is the gas pressure in torr, $A$ is the electrode area, $k$ is the ion mobility constant for the used gas and $d_{S}$ is the sheath thickness given by the following equation: 


$$
d_{S}=\omega \varepsilon A\left|X_{p}\right|
$$

where $X_{p}$ is capacitive reactance of the sheath which given by

$$
\left|X_{p}\right|=\frac{\left|V_{p}\right|}{I_{m}}, \quad I_{m}=\sqrt{2} I
$$

where $I$ is discharge current.

After rearrangement the equations one yields;

$$
\begin{aligned}
& P_{S}=\left(\left|V_{p}\right|\left|I_{R}\right|\right)^{5 / 3} \frac{3 \varepsilon_{0} A}{5}\left(\frac{3 \sqrt{p}}{2 k}\right)^{2 / 3}\left(\frac{1}{\sqrt{2}}\right)^{5 / 3}\left(\frac{\omega}{I}\right)^{5 / 3} \\
& =\left(P_{S}\right)^{5 / 3} \frac{3 \varepsilon_{0} A}{5}\left(\frac{3 \sqrt{p}}{2 k}\right)^{2 / 3}\left(\frac{1}{\sqrt{2}}\right)^{5 / 3}\left(\frac{\omega}{I}\right)^{5 / 3}
\end{aligned}
$$

After solving for $P_{S}$, one can obtain the final expression for the power dissipated in the sheath as follows:

$$
P_{S}=(\sqrt{2})^{5 / 2}\left(\frac{5}{3 \varepsilon_{0} A}\right)^{3 / 2}\left(\frac{2 k}{3 \sqrt{p}}\right)\left(\frac{I}{\omega}\right)^{5 / 2}
$$

\subsection{Sheath Thickness}

The sheath thickness $d_{s}$ is defined by the capacitor formula $C_{s}=\varepsilon_{0} \mathrm{~A} / d_{s}$, where $C_{s}$ is the sheath capacitance.

Relating $C_{s}$ to $X$ from the relation

$$
\begin{gathered}
X=-1 / \omega C_{s} \\
\therefore \quad d_{s}=\omega \varepsilon_{0} A|X| \\
|X|=V_{R F} / I
\end{gathered}
$$

where $V_{R F}$ is the $R F$ voltage across the tube and $I$ is the discharge current.

At $V_{R F}=930 \mathrm{~V}, I=20 \mathrm{~mA}$ and $A=3 \times 10^{-2} \mathrm{~m}^{-2}$, then $d_{s}$ is of the order of $0.01 \mathrm{~m}$.

According to the description given by Allen et al. [14] the sheath thickness $d_{s}$ of a capacitively coupled $R F$ discharge should be of the same order of the electron oscillation amplitude $X_{m}$ in the $R F$ field of the discharge.

$$
\therefore \quad d_{s} \approx X_{m} .
$$

Those authors give the plausible argument that the maximum distance the electrons can be displaced during a half cycle is equal to the sheath thickness; otherwise they would be captured by the electrodes.

\section{Results and Discussion}

The relation between the applied voltage " $V$ " and the discharge current " $I$ " of the $R F$ discharge is measured. The measurements have been carried out in the pressure range (0.5 - 2 torr) for Ar gas. $I-V$ characteristic curves for Ar gas at pressures of 0.5, 1, 1.5, and 2 torr are shown in Figure 3.

The relation between the applied voltage and the discharge current can be explained as the following:

When the applied voltage increased the amplitude of the $R F$ electric field is increased, so, the oscillating electrons gain enough energy to make collisions with the neutral particles and increasing the ionization rate, consequently the discharge current would increase. At high pressure, the number of electrons-atom ionizing collision increases, thus, more electrons are produced and consequently, the discharge current is increased. Moreover, the voltage across the plasma sheath cannot be specified independently of the heating mechanism and the power absorbed by the plasma.

On the other hand, the self-consistent model is used for high pressure capacitive $R F$ discharge. Assuming the following: 1) The only loss mechanism is electron loss to the wall; 2) The ionization occurs in the sheath, and the electrons are accelerated into the plasma; 3) The plasma length $d \approx 1-2 S_{m}$ (where $S_{m}$ is the maximum sheath thickness) $l$ is the system length, with an initial estimate $S_{m} \approx 1 \mathrm{~cm}$ for numerical computations which is a no- 


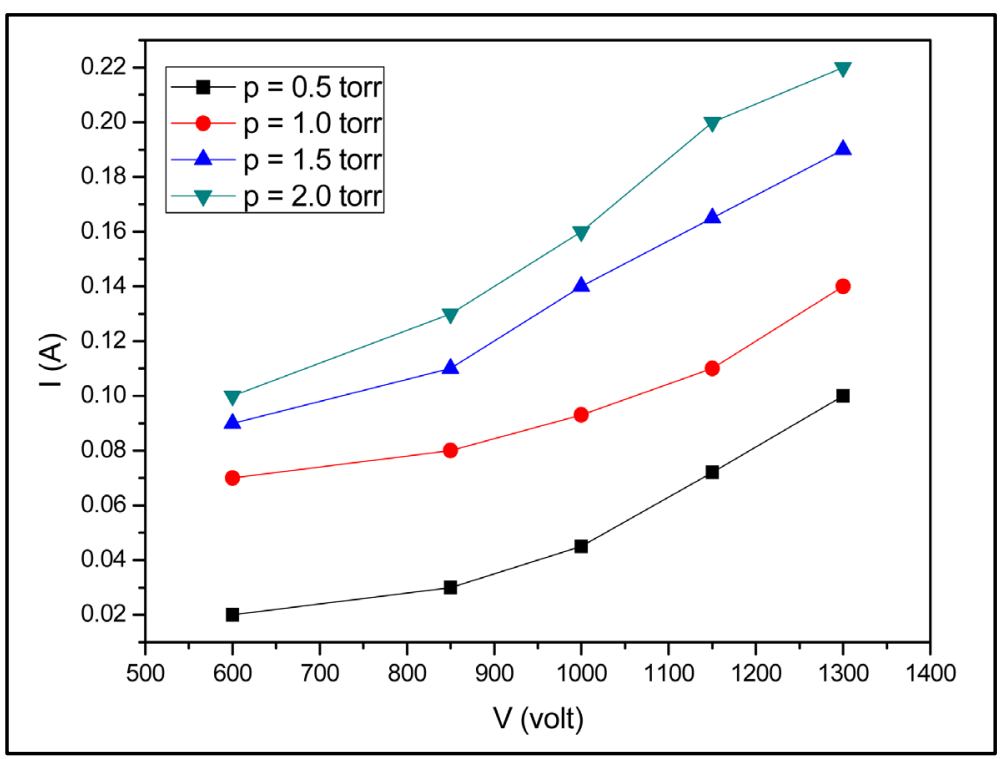

Figure 3. The $I-V$ characteristic curves for Ar gas at different pressures.

minal value for high-pressure capacitive $R F$ discharge.

The electron ohmic heating power per unit area and the average Stochastic power for a single sheath are calculated using Equations (7) and (8) respectively. The calculation results indicate that, at high pressure, the ohmic heating is usually the dominant heating mechanism. Ohmic heating due to the transfer of energy gained from the acceleration of electrons in electric field to thermal electron energy through collisional processes [14].

The power dissipated in the sheath $P_{s}$ is calculated using Equation (18), (where the constant $k$ for Ar gas is equal to 103.75 [13]. Figure 4 shows the comparison between the measured dissipated power $P_{s}$, measured using the wattmeter, and the calculated one as a function of Ar gas pressure at constant discharge current $I=20$ $\mathrm{mA}$. When the gas pressure increased the dissipated power is decreased. At high gas pressure, the secondary electrons traversing the near-electrode sheath have an opportunity to experience one or several elastic and/or inelastic collisions that results in lesser energy acquired by them over the sheath width compared with the case without collisions.

Moreover, Figure 5 shows the relation between the input power of the discharge, which calculated from the measured waveform of the discharge currents, at two constant discharge voltage, as a function of the gas pressure. When the gas pressure increases the gas discharge is more confined in the electrode space due to the lower mean free path associated with a large number of collisions. It's clear, however, that one can vary the power consumed by the discharge not only by varying the discharge voltage $V$ but also by varying the chamber pressure at a constant $V$.

In contrast to the measurements presented, the total power measured by the wattmeter, before the matching network, shows the small variations of the order of $\pm 10 \%$ when increasing the gas pressure, while the power really consumed in the discharge changes more than that for higher applied voltage. The reason for that is, most of the power output of the power supply is consumed in the matching network and the stray impedance of the system. Thus a relation between the actual dissipated power $P_{s}$ in the discharge to the total power $P_{T}$ measured by the wattmeter which include, the power consumed in the discharge and the power losses in the matching network, is shown in Figure 6. The actual power is a small non constant fraction of the total power and the amount of power consumed in the discharge is higher at higher applied voltages and gas pressures. This is due to the fact that plasma impedance is changing and thus a large amount of power is transferred from the power supply to the discharge [15].

Moreover, the sheath thickness $d_{s}$ of a capacitively coupled $R F$ discharge is calculated using Equation (19), $d_{s}$ should be of the same order of the electron oscillation amplitude $X_{m}$ in the $R F$ field of the discharge. The higher sheath field will increase the amount of power consumed for the acceleration of ions and secondary electrons in the sheath. In the case of higher pressures, the smaller mean free path of the electrons leads to smaller sheath dimensions and thus to higher sheath capacitance [16]. 


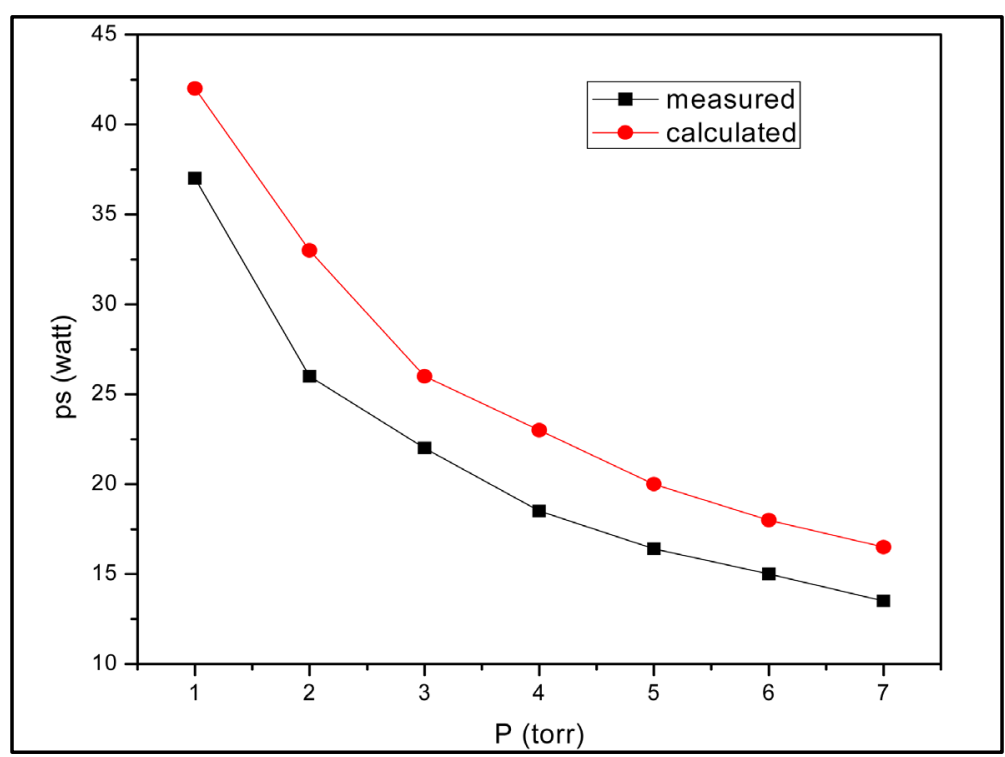

Figure 4. It shows the comparison between the measured dissipated power $P_{s}$, measured using the wattmeter, and the calculated one as a function of $\mathrm{Ar}$ gas pressure at constant discharge current $I=20 \mathrm{~mA}$.

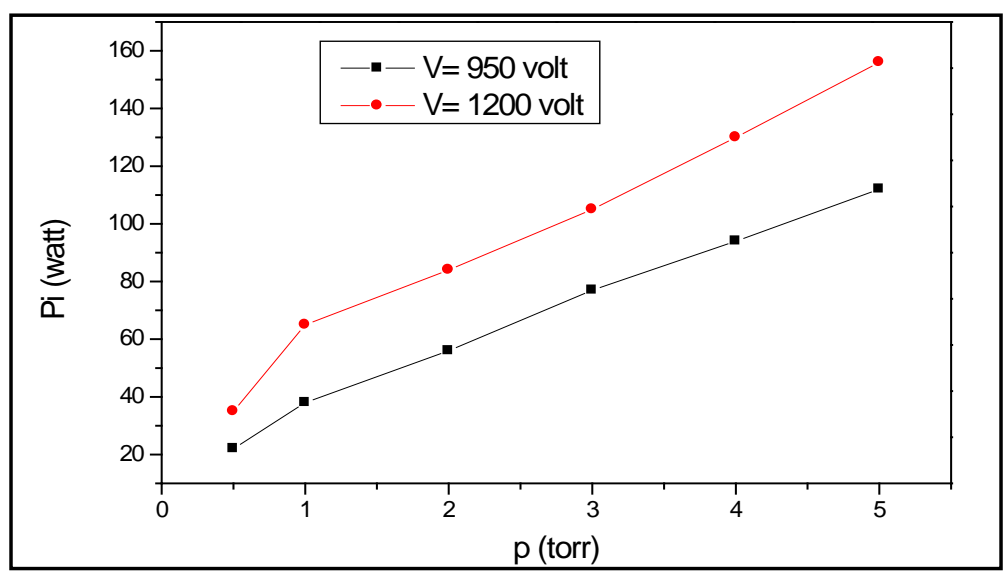

Figure 5. It shows the relation between the input power of the discharge, which calculated from the measured waveform of the discharge currents, at two constant discharge voltage, as a function of the gas pressure.

\section{Conclusions}

A self-consistent model of the collisional sheath of the capacitively coupled $R F$ discharge at high gas pressure is used. The electron ohmic heating power, the average Stochastic power for a single sheath, the power dissipated in the sheath and the sheath thickness are calculated.

The plasma is produced, using $13.65 \mathrm{MHz} R F$ source, using Ar gas. The relation between the applied voltage " $V$ " and the discharge current " $I$ " of the $R F$ discharge is measured. The voltage across the plasma sheath cannot be specified independently of the heating mechanism and the power absorbed by the plasma. The calculation results indicate that, at high pressure, the ohmic heating is usually the dominant heating mechanism. The electrons are heated by ohmic heating resulting from the volume currents, rather than from sheath heating.

At constant discharge current $I=20 \mathrm{~mA}$, the calculated and/or measured dissipated power in the sheath is decreased when the gas pressure is increased.

The relation between the actual dissipated power $P_{s}$ in the discharge to the total power $P_{T}$ measured by the wattmeter which include, the power consumed in the discharge and the power losses in the matching network, is 


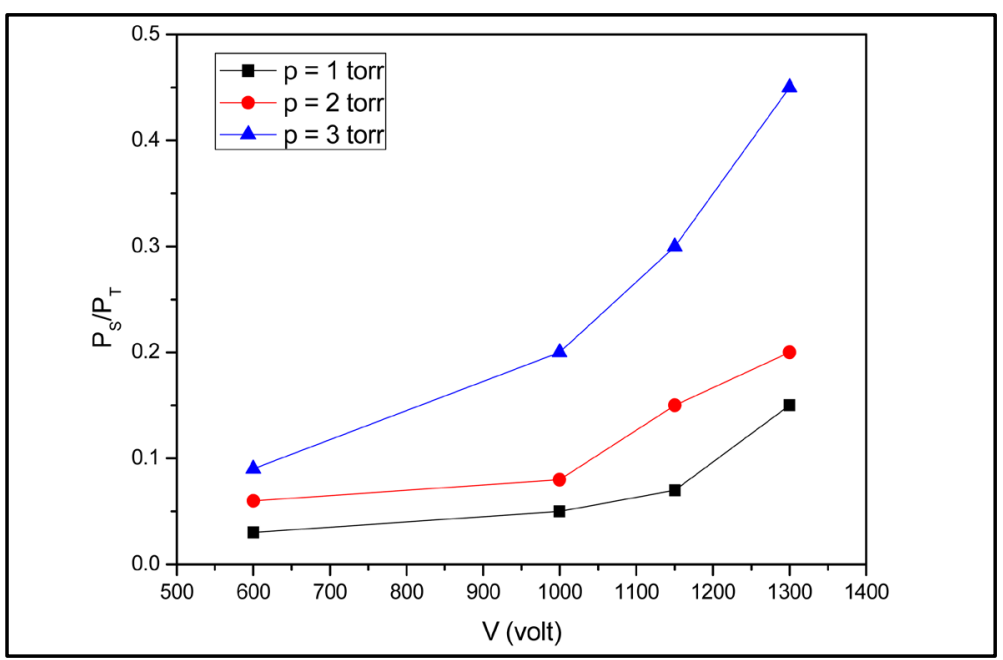

Figure 6. The relation between the ratio $\left(P_{S} / P_{T}\right)$ as a function of the discharge voltage $V$ of the discharge voltage $\mathrm{V}$ at different gas pressures

given. The actual power is a small non-constant fraction of the total power and the amount of power consumed in the discharge is higher at higher applied voltages and gas pressures. The power coupling to the discharge is sensitive to the variation of both the discharge voltage and gas pressure.

Moreover, the sheath thickness $d_{s}$ of a capacitively coupled $R F$ discharge is calculated and found to be of the same order of the electron oscillation amplitude $X_{m}$ in the $R F$ field of the discharge.

\section{References}

[1] Galaly, A.R. and El Akshar, F.F. (2013) International Journal of Multidisciplinary Research and Advances in Engineering (IJMRAE), 5, 169-179.

[2] Galaly, A.R., Elakshar, F.F. and Khedr, M.A. (2013) Materials Science Forum, 756, 143-150. http://dx.doi.org/10.4028/www.scientific.net/MSF.756.143

[3] Galaly, A.R. and Elakshar, F.F. (2013) Journal of Modern Physics, 4, 215-225. http://dx.doi.org/10.4236/jmp.2013.42030

[4] Hassouba, M.A., Nossair, A.M. and El-Akashar, F.F. (2001) Jurnal Fizik Malaysia, 22, 1.

[5] Lisovskiy, V.A. and Yegorenkov, V.D. (2004) Vacuum, 74, 19-28. http://www.sciencedirect.com/science/journal/0042207X/74/1 http://dx.doi.org/10.1016/j.vacuum.2003.11.003

[6] Chen, F.F. (2006) Plasma Sources Science and Technology, 15, 773. http://dx.doi.org/10.1088/0963-0252/15/4/022

[7] Chen, F.F. (2009) Plasma Sources Science and Technology, 18, Article ID: 035012. http://dx.doi.org/10.1088/0963-0252/18/3/035012

[8] Amanatides, E. and Mataras, D. (2001) Journal of Applied Physics, 89, 1556. http://dx.doi.org/10.1063/1.1337597

[9] Dudnikov, V. and Dudnikov, A. (2012) Review of Scientific Instruments, 83, Article ID: 02A720.

[10] Godyak, V.A., Piejak, R.B. and Alexandrovich, B.M. (1991) IEEE Transactions on Plasma Science, 19, 660-676. http://dx.doi.org/10.1109/27.90309

[11] Liebermann, M.A. (1989) Journal of Applied Physics, 65, 4168. http://dx.doi.org/10.1063/1.343298

[12] Godyak, V.A. (1990) IEEE Transactions on Plasma Science, 18 159-168. http://dx.doi.org/10.1109/27.45519

[13] Liebermann, M.A. and Lichtenberg, A.J. (1994) Principles of Plasma Discharges and Materials Processes. John Wiley \& Sons Inc., New York.

[14] Allen, J.A., Boyd, R.L. and Reynolds, P. (1957) Proceedings of the Physical Society B, 70, 297. http://dx.doi.org/10.1088/0370-1301/70/3/303

[15] Surendra, M. and Graves, D.B. (1990) Applied Physics Letters, 56, 1022. http://dx.doi.org/10.1063/1.102604

[16] Spiliopoulos, N., Mataras, D. and Rapakoulias, D.E. (1996) Journal of Vacuum Science \& Technology A, $14,2757$. http://dx.doi.org/10.1116/1.580197 